\title{
GAIT RECOGNITION THROUGH MPCA PLUS LDA
}

\author{
Haiping Lu, K.N. Plataniotis and A.N. Venetsanopoulos \\ The Edward S. Rogers Sr. Department of Electrical and Computer Engineering \\ University of Toronto, M5S 3G4, Canada \\ \{haiping, kostas,anv\}@dsp.toronto.edu
}

\begin{abstract}
This paper solves the gait recognition problem in a multilinear principal component analysis (MPCA) framework. Gait sequences are naturally described as tensor objects and feature extraction for tensor objects is important in computer vision and pattern recognition applications. Classical principal component analysis (PCA) operates on vectors and it is not directly applicable to gait sequences. This work introduces an MPCA framework for feature extraction from gait sequences by seeking a multilinear projection onto a tensor subspace of lower dimensionality which captures most of the variance of the original gait samples. A subset of the extracted eigentensors are selected and the classical LDA is then applied. In experiments, gait recognition results are reported on the Gait Challenge data sets using the proposed solution. The results indicate that with a simple design, the proposed algorithm outperforms the state-of-the-art algorithms.
\end{abstract}

\section{INTRODUCTION}

Gait recognition [1,2], the identification of individuals in video sequences by the way they walk, has recently gained significant attention. This interest is strongly motivated by the need for automated person identification system at a distance in visual surveillance and monitoring applications in securitysensitive environments, e.g., banks, parking lots, and airports, where other biometrics such as fingerprint, face or iris information are not available at high enough resolution for recognition [3]. Furthermore, night vision capability is usually impossible with other biometrics due to the limited signature in the IR image [3]. Gait is a complex spatio-temporal biometric characteristic that can address the problems above.

Most of the gait recognition algorithms proposed in the literature take gait silhouette sequences as the input, which are three-dimensional objects naturally represented as thirdorder tensors, with the spatial row, column and the temporal modes for the three dimensions. They are specified in a very

The authors would like to thank Prof. Sarkar from the USF for providing the Gait Challenge data sets. Support provided by the Centre of Excellence of the Province of Ontario through its partnership Program and the Bell University Labs - at the University of Toronto is also acknowledged. high-dimensional tensor space and recognition methods operating directly on this space suffer from the curse of dimensionality [4]. However, the entries of gait silhouette sequences are often highly correlated with surrounding entries and these gait sequences are highly constrained. They will in fact be confined to a gait (tensor) subspace.

Dimensionality reduction is commonly used to transform a high-dimensional data set into a low-dimensional subspace while retaining most of the underlying structure in the data [5]. Principal Component Analysis (PCA) is a classical linear technique for dimensionality reduction but for direct application on gait sequences, it needs to reshape (vectorize) these tensor objects into vectors in a very high-dimensional space, resulting in high computation and memory demand. Furthermore, the reshaping breaks the structure and correlation in the original data and thus the redundancy and structure in the original data is not fully preserved. Therefore, a dimensionality reduction algorithm operating directly on the gait sequences in their tensor representation rather than their vectorized versions is desirable. Nevertheless, this approach is not well researched and most of the gait recognition algorithms in the literature extract features based on frame-byframe processing and they are not fully utilizing the spatial and temporal correlations in the data $[1,3,6]$. Recently, an attempt has been made to solve the gait recognition problem by extending PCA to the multilinear case [7], where higher order SVD (HOSVD) [8] truncation has been used.

In this paper, as a further development of [7], a new multilinear principal component analysis (MPCA) formulation is proposed for dimensionality reduction and feature extraction in gait recognition. Operating directly on the original gait data in their tensorial representation, the proposed MPCA is a multilinear algorithm performing dimensionality reduction in all modes by seeking a number of bases in each mode that allows most of the variations in the original gait samples to be captured by the projected features. Section 2 presents the new MPCA framework and in Section 3, an MPCA-based gait recognition system that performs LDA on selected gait features obtained directly from gait samples represented as tensor objects is described. Experimental results on gait recognition are presented in Section 4 and it is shown that the proposed algorithm outperforms the state-of-the-art gait recogni- 
tion algorithms. Finally, Section 5 concludes the paper and discusses future works.

\section{THE MPCA FRAMEWORK}

An $N^{t h}$-order tensor $\mathcal{X}$ is in the tensor (multilinear) space $\mathbb{R}^{I_{1}} \otimes \mathbb{R}^{I_{2}} \ldots \otimes \mathbb{R}^{I_{N}}$, where $\otimes$ denotes the Kronecker product and $\mathbb{R}^{I_{1}}, \mathbb{R}^{I_{2}}, \ldots, \mathbb{R}^{I_{N}}$ are the $N$ vector (linear) spaces. For gait sequences, the corresponding tensor space is very highdimensional and a class of gait sequences typically are embedded in a tensor subspace (or manifold). Thus, a tensor subspace can be found so that it captures most of the variations in the collection of the original gait samples and it can be used to extract features for applications such as gait recognition. To achieve this objective, $P_{n}<I_{n}$ orthonormal basis vectors (principle axes) of the $n$-mode linear space $\mathbb{R}^{I_{n}}$ are sought for each $n$ and a tensor subspace $\mathbb{R}^{P_{1}} \otimes \mathbb{R}^{P_{2}} \ldots \otimes \mathbb{R}^{P_{N}}$ is formed by these linear subspaces. Let $\tilde{\mathbf{U}}^{(n)}$ denote the $I_{n} \times P_{n}$ matrix containing the $P_{n}$ orthornormal $n$-mode basis vectors. The projection of $\mathcal{X}$ onto the tensor subspace $\mathbb{R}^{P_{1}} \otimes \mathbb{R}^{P_{2}} \ldots \otimes \mathbb{R}^{P_{N}}$ is defined as $\mathcal{Y}=\mathcal{X} \times{ }_{1} \tilde{\mathbf{U}}^{(1)^{T}} \times{ }_{2} \tilde{\mathbf{U}}^{(2)^{T}} \ldots \times{ }_{N} \tilde{\mathbf{U}}^{(N)^{T}}$.

\subsection{The MPCA algorithm}

In this section, a multilinear principal component analysis (MPCA) solution to the problem of dimensionality reduction for gait sequences is introduced based on the analysis introduced in [9]. The problem to be solved is formally stated as follows:

A set of $M$ training gait samples $\left\{\mathcal{X}_{1}, \mathcal{X}_{2}, \ldots, \mathcal{X}_{M}\right\}$ is available. Each gait sample $\mathcal{X}_{m} \in \mathbb{R}^{I_{1} \times I_{2} \times \ldots \times I_{N}}$ assumes values in the tensor space $\mathbb{R}^{I_{1}} \otimes \mathbb{R}^{I_{2}} \ldots \otimes \mathbb{R}^{I_{N}}$, where $I_{n}$ is the $n$-mode dimension of the gait tensor. The objective of MPCA is to find a multilinear transformation $\left\{\tilde{\mathbf{U}}^{(n)} \in\right.$ $\left.\mathbb{R}^{I_{n} \times P_{n}}, n=1, \ldots, N\right\}$ mapping from the original gait tensor space $\mathbb{R}^{I_{1}} \otimes \mathbb{R}^{I_{2}} \ldots \otimes \mathbb{R}^{I_{N}}$ into a tensor subspace $\mathbb{R}^{P_{1}} \otimes \mathbb{R}^{P_{2}}$ $\ldots \otimes \mathbb{R}^{P_{N}}$ (with $P_{n}<I_{n}$, for $\left.n=1, \ldots, N\right): \mathcal{Y}_{m}=\mathcal{X}_{m} \times_{1}$ $\tilde{\mathbf{U}}^{(1)^{T}} \times{ }_{2} \tilde{\mathbf{U}}^{(2)^{T}} \ldots \times_{N} \tilde{\mathbf{U}}^{(N)^{T}}, m=1, \ldots, M$, such that $\left\{\mathcal{Y}_{m} \in\right.$ $\left.\mathbb{R}^{P_{1}} \otimes \mathbb{R}^{P_{2}} \ldots \otimes \mathbb{R}^{P_{N}}, m=1, \ldots, M\right\}$ captures as much as possible the variations in the original gait samples, where the variations are measured by the total scatter defined below.

Definition 1. Let $\left\{\mathcal{A}_{m}, m=1, \ldots, M\right\}$ be a set of $M$ gait samples in $\mathbb{R}^{I_{1}} \otimes \mathbb{R}^{I_{2}} \ldots \otimes \mathbb{R}^{I_{N}}$. The total scatter of these samples is defined as: $\Psi_{\mathcal{A}}=\sum_{m=1}^{M}\left\|\mathcal{A}_{m}-\overline{\mathcal{A}}\right\|_{F}^{2}$, where $\overline{\mathcal{A}}$ is the mean sample calculated as $\overline{\mathcal{A}}=\frac{1}{M} \sum_{m=1}^{M} \mathcal{A}_{m}$.

Based on Definition 1, the objective function of MPCA is to determine the $N$ projection matrices $\left\{\tilde{\mathbf{U}}^{(n)} \in \mathbb{R}^{I_{n} \times P_{n}}, n=\right.$ $1, \ldots, N\}$ that maximize the total tensor scatter $\Psi_{\mathcal{Y}}$ :

$$
\left\{\tilde{\mathbf{U}}^{(n)}, n=1, \ldots, N\right\}=\arg \max _{\tilde{\mathbf{U}}^{(1)}, \tilde{\mathbf{U}}^{(2)}, \ldots, \tilde{\mathbf{U}}^{(N)}} \Psi_{\mathcal{Y}} .
$$

Here, the dimensionality $P_{n}$ for each mode is assumed to be known or pre-determined. Discussions on how to determine $P_{n}$ when it is not given will be presented later.
Unfortunately, there is no known optimal solution to simultaneously optimize the $N$ projection matrices. Since the projection to an $N^{t h}$-order tensor subspace can be decomposed into $N$ projections to $N$ vector subspaces, $N$ optimization subproblems can be obtained by finding each $\tilde{\mathbf{U}}^{(n)}$ maximizing the scatter in the $n$-mode vector subspaces.

The following theorem provides the theoretical fundamentals for the framework introduced. The proof of the theorem and additional information, such as the relationship with PCA and 2DPCA, can be found in [9].

Theorem 1. Let $\left\{\tilde{\mathbf{U}}^{(n)}, n=1, \ldots, N\right\}$ be the solution to Equation (1). Then, for given $\tilde{\mathbf{U}}^{(1)}, \ldots, \tilde{\mathbf{U}}^{(n-1)}, \tilde{\mathbf{U}}^{(n+1)}, \ldots$, $\tilde{\mathbf{U}}^{(N)}$, the matrix $\tilde{\mathbf{U}}^{(n)}$ consists of the $P_{n}$ eigenvectors corresponding to the largest $P_{n}$ eigenvalues of the matrix

$\Phi^{(n)}=\sum_{m=1}^{M}\left(\mathbf{X}_{m(n)}-\overline{\mathbf{X}}_{(n)}\right) \cdot \tilde{\mathbf{U}}_{\Phi^{(n)}} \cdot \tilde{\mathbf{U}}_{\Phi^{(n)}}^{T} \cdot\left(\mathbf{X}_{m(n)}-\overline{\mathbf{X}}_{(n)}\right)^{T}$,

where $\tilde{\mathbf{U}}_{\Phi^{(n)}}=\left(\tilde{\mathbf{U}}^{(n+1)} \otimes \ldots \otimes \tilde{\mathbf{U}}^{(N)} \otimes \tilde{\mathbf{U}}^{(1)} \otimes \ldots \tilde{\mathbf{U}}^{(n-1)}\right)$.

Since $\tilde{\mathbf{U}}_{\boldsymbol{\Phi}^{(n)}} \cdot \tilde{\mathbf{U}}_{\boldsymbol{\Phi}^{(n)}}^{T}$ depends on $\tilde{\mathbf{U}}^{(1)}, \ldots, \tilde{\mathbf{U}}^{(n-1)}, \tilde{\mathbf{U}}^{(n+1)}$, $\ldots, \tilde{\mathbf{U}}^{(N)}$, the optimization of $\tilde{\mathbf{U}}^{(n)}$ depends on the projections in other modes and there is no closed form solution to this maximization problem. Instead, an iterative solution was introduced by Theorem 1: The input gait samples (as tensors) are centered and properly initialized, the projection matrices are computed one by one with all the others fixed (local optimization) according to Theorem 1. The local optimization procedure can be repeated until convergence with each iteration guaranteeing an improvement of the objective function $\Psi_{\mathcal{Y}}$, in a similar fashion as the alternating least-squares (ALS) method [10].

In the following, several issues related to the application of the MPCA algorithm to the problem of gait feature extraction are discussed: the initialization, the termination criteria, the determination of the subspace dimensionality and the computational complexity.

\subsection{Initialization and termination}

Full projection truncation (FPT) is used to initialize the MPCA iteration. Here, the term full projection refers to the multilinear projection for MPCA with $P_{n}=I_{n}$ for $n=1, \ldots, N$, and in this case, $\mathbf{U}_{\boldsymbol{\Phi}^{(n)}} \cdot \mathbf{U}_{\boldsymbol{\Phi}^{(n)}}^{T}$ is an identity matrix and $\boldsymbol{\Phi}^{(n)}$ is reduced to $\boldsymbol{\Phi}^{(n) *}=\sum_{m=1}^{M}\left(\mathbf{X}_{m(n)}-\overline{\mathbf{X}}_{(n)}\right) \cdot\left(\mathbf{X}_{m(n)}-\overline{\mathbf{X}}_{(n)}\right)^{T}$. Now $\boldsymbol{\Phi}^{(n) *}$ is determined by the input gait samples only and is independent on other projection matrices. The optimal $\tilde{\mathbf{U}}^{(n)}=$ $\mathbf{U}^{(n)}$ is then obtained as the eigenvectors of $\boldsymbol{\Phi}^{(n) *}$ directly without iteration, and the total scatter $\Psi_{\mathcal{X}}$ in the original data is fully captured but dimensionality is not reduced. By writing the projection in the form of (4), each gait sample in the class can then be written as a linear combination of $P_{1} \times P_{2} \times$ $\ldots \times P_{N}$ rank-1 tensors $\tilde{\mathcal{U}}_{p_{1} p_{2} \ldots p_{N}}=\tilde{\mathbf{u}}_{p_{1}}^{(1)} \circ \tilde{\mathbf{u}}_{p_{2}}^{(2)} \circ \ldots \circ \tilde{\mathbf{u}}_{p_{N}}^{(N)}$, which are named as eigentensors in this paper. 
Keeping the first $P_{n}$ columns of the full projection matrix $\mathbf{U}^{(n)}$ in $n$-mode for all $n$ gives an initial projection matrix $\tilde{\mathbf{U}}^{(n)}$. This solution is close to the (local) optimal solution and it is a good point to start the iterations in MPCA. This initialization is equivalent to the MPCA solution based on HOSVD truncation in [7].

The termination criterion is to be determined using the objective function $\Psi_{\mathcal{Y}}$. In particular, the iterative procedure is terminated if $\left(\Psi_{\mathcal{Y}_{k}}-\Psi_{\mathcal{Y}_{k-1}}\right)<\eta$, where $\Psi_{\mathcal{Y}_{k}}$ and $\Psi_{\mathcal{Y}_{k-1}}$ are the results from the $k^{t h}$ and $(k-1)^{t h}$ iterations, respectively. In addition, the maximum number of iterations allowed is set to $K$ for computational consideration.

\subsection{Determination of the subspace dimensionality}

When $P_{n}$ for each $n$ is not specified, a method named $Q$ based method is proposed to determine them. From the definition of the Frobenius norm, $\|\mathcal{A}\|_{F}=\left\|\mathbf{A}_{(n)}\right\|_{F}$. Thus, the total scatter for full projection $\Psi_{\mathcal{Y}}^{*}=\Psi_{\mathcal{X}}=\sum_{m=1}^{M} \|$ $\mathbf{Y}_{m(n)}-\overline{\mathbf{Y}}_{(n)} \|_{F}^{2}=\sum_{i_{n}=1}^{I_{n}} \lambda_{i_{n}}^{(n) *}, n=1, \ldots, N$, where $\lambda_{i_{n}}^{(n) *}$ is the $i_{n}^{t h} n$-mode eigenvalue (thus $\sum_{i_{1}=1}^{I_{1}} \lambda_{i_{1}}^{(1) *}=\sum_{i_{2}=1}^{I_{2}} \lambda_{i_{2}}^{(2) *}$ $\left.=\ldots=\sum_{i_{N}=1}^{I_{N}} \lambda_{i_{N}}^{(N) *}=\Psi_{\mathcal{X}}\right)$. Truncation of the $n$-mode eigenvectors after $P_{n}$ discards a portion of $\frac{\sum_{i_{n}=P_{n}+1}^{I_{n}} \lambda_{i_{n}}^{(n) *}}{\sum_{i_{n}=1}^{I_{n}} \lambda_{i_{n}}^{(n)} *}$ of the total scatter in the $n$-th mode. Define the ratio of variations kept in the $n$-mode as $Q^{(n)}=\frac{\sum_{i_{n}=1}^{P_{n}} \lambda_{i_{n}}^{(n) *}}{\sum_{i_{n}=1}^{I n} \lambda_{i_{n}}^{(n) *}}$. In the proposed method, the first $P_{n}$ eigenvectors are kept in each mode so that the same (or similar) amount of variances (energy) is kept in each mode: $Q^{(1)}=Q^{(2)}=\ldots=Q^{(N)}=Q$. This strategy is an extension of the dimensionality selection strategy in PCA to the multilinear case. The reason behind this choice is that the loss of variations due to dimensionality reduction is approximately proportional to the sum of the corresponding eigenvalues of the discarded eigenvectors. Thus, the variation loss resulted from discarding the least significant eigenvectors in each mode is low and truncation of least significant eigenvectors in all modes is advantageous.

\subsection{Discussion on computational complexity}

The time complexity for one iteration is studied. It is assumed that $I_{1}=I_{2}=\ldots=I_{N}=\left(\prod_{n=1}^{N} I_{n}\right)^{\frac{1}{n}}=I$ for simplicity. The most time-consuming steps are the formation of the matrices $\boldsymbol{\Phi}^{(n)}$, the eigen-decomposition of $\boldsymbol{\Phi}^{(n)}$, and the computation of the multilinear projection $\mathcal{Y}_{m} \cdot \boldsymbol{\Phi}^{(n)}$ is more efficiently computed using multilinear multiplication rather than Kronecker products. The computations of $\boldsymbol{\Phi}^{(n)}$, the $P_{n}$ eigenvectors of $\boldsymbol{\Phi}^{(n)}$ and $\mathcal{Y}_{m}$ take $O\left(M N \cdot I^{(N+1)}\right)$ (upper bounded), $O\left(I^{3}\right)$ and $O\left(N \cdot I^{(N+1)}\right)$, respectively. The total complexity is $O\left((N+1) \cdot M N \cdot I^{(N+1)}+N * I^{3}\right)$.

In MPCA, $\overline{\mathcal{X}}$ and $\boldsymbol{\Phi}^{(n)}$ can be computed incrementally by reading $\mathcal{X}_{m}$ or $\tilde{\mathcal{X}}_{m}$ sequentially without loss of informa- tion. Hence, memory requirements for the MPCA algorithm can be as low as $O\left(\prod_{n=1}^{N} I_{n}\right)$ and MPCA computes the solution without requiring all gait samples in the memory. This is a major advantage of MPCA, especially for large databases, compared with approaches forming an $(N+1)^{t h}$-order tensor for $N^{t h}$-order tensor gait samples to perform HOSVD [7], which imposes very high memory requirement and increases the time complexity as well.

\section{GAIT RECOGNITION USING MPCA PLUS LDA}

In the problem of gait recognition, the input data are gait image sequences with very high-dimensionality. The projection matrices $\left\{\tilde{\mathbf{U}}^{(n)}, n=1, \ldots, N\right\}$ obtained through maximizing $\Psi_{\mathcal{Y}}$ of a set of training gait samples $\left\{\mathcal{X}_{m}, m=1, \ldots, M\right\}$ through MPCA can be used on these sequences in their natural tensor representation for dimensionality reduction and feature extraction. The gait recognition system through MPCA plus LDA is depicted in Fig. 1. As in other biometric recognition problems, such as human identification using fingerprints or faces, there are two types of gait data sets: the gallery and the probe [1]. The gallery set contains the set of gait samples with known identities and it is used as the training set. The probe set is the testing set with gait samples of unknown identities to be identified through matching against the gallery set.

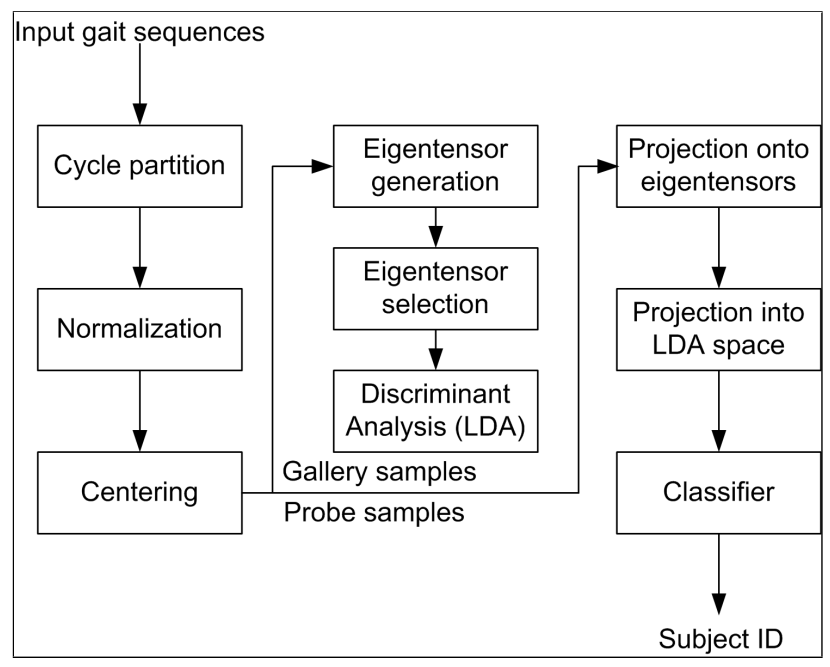

Fig. 1. MPCA plus LDA for gait recognition.

An input gait sequence is firstly partitioned into a number of gait samples. As in [7], each half gait cycle is treated as a gait sample (3rd-order tensor) in this paper, which is obtained through counting the number of foreground pixels in the bottom half of each silhouette. This number sequence is smoothed with a running average filter and the minimums in this sequence partition the sequence into several half gait cycles. 
Since MPCA takes samples of the same dimensions in all the modes for feature extraction, the input gait samples (half cycles) need to be normalized to standard dimensions first. The spatial (row and column) modes normalization is through image resizing and the time mode normalization is through temporal interpolation as in [7]. The normalized tensor samples are then centered by subtracting the mean obtained from the gallery samples.

From the gallery set, a set of eigentensors are obtained, with $P_{n}$ determined by a user-specified $Q$. For the task of gait recognition, it should be noted that although with $P_{n}<I_{n}$, some of the small variations and noise are removed but the remaining eigentensors encode not only the inter-subject variations but also the intra-subject variations in the gallery set. Clearly not all of them are useful for recognition and a feature selection strategy is proposed to select eigentensors according to their class discriminability [11], which is defined as the ratio of the between-class and within-class scatter, following the framework of [9]:

Definition 2. The class discriminability $\Gamma_{p_{1} p_{2} \ldots p_{N}}$ for the eigentensor $\tilde{\mathcal{U}}_{p_{1} p_{2} \ldots p_{N}}$ is defined as

$\Gamma_{p_{1} p_{2} \ldots p_{N}}=\frac{\sum_{c=1}^{C} N_{c} \cdot\left[\overline{\mathcal{Y}}_{c}\left(p_{1}, p_{2}, \ldots, p_{N}\right)-\overline{\mathcal{Y}}\left(p_{1}, p_{2}, \ldots, p_{N}\right)\right]^{2}}{\sum_{m=1}^{M}\left[\mathcal{Y}_{m}\left(p_{1}, p_{2}, \ldots, p_{N}\right)-\overline{\mathcal{Y}}_{c_{m}}\left(p_{1}, p_{2}, \ldots, p_{N}\right)\right]^{2}}$,

where $C$ is the number of classes (subjects), $M$ is the total number of gait samples in the gallery set, $N_{c}$ is the number of gait samples for class (subject) $c$ and $c_{m}$ is the class label for the $m^{\text {th }}$ gallery gait sample $\mathcal{X}_{m} . \mathcal{Y}_{m}$ is the feature tensor of $\mathcal{X}_{m}$ in the projected tensor subspace, the mean feature tensor $\overline{\mathcal{Y}}=\frac{1}{M} \sum_{m} \mathcal{Y}_{m}$ and the class mean feature tensor $\overline{\mathcal{Y}}_{c}=$ $\frac{1}{N_{c}} \sum_{m, c_{m}=c} \mathcal{Y}_{m}$.

For the selection, the entries in $\mathcal{Y}_{m}$ are arranged into a feature vector $\mathbf{y}_{m}$ ordered according to $\Gamma_{p_{1} p_{2} \ldots p_{N}}$ in descending order and only the first $H_{\mathbf{y}}$ entries of $\mathbf{y}_{m}$ are kept for classification, with $H_{\mathbf{y}}$ determined empirically or user-specified.

A classical linear discriminant analysis (LDA) is then applied to obtain an MPCA+LDA approach for recognition, similar to the popular approach of PCA+LDA [12]. LDA seeks a projection $\mathbf{V}$ to maximize the ratio of the between-class scatter matrix $\mathbf{S}_{B}$ to the within-class scatter matrix $\mathbf{S}_{W}$, where $\mathbf{S}_{W}=\sum_{m}\left(\mathbf{y}_{m}-\overline{\mathbf{y}}_{c_{m}}\right)\left(\mathbf{y}_{m}-\overline{\mathbf{y}}_{c_{m}}\right)^{T}$ and $\overline{\mathbf{y}}_{c}=\frac{1}{N_{c}} \sum_{m}^{c_{m}=c} \mathbf{y}_{m}$, $\mathbf{S}_{B}=\sum_{c=1}^{C} N_{c}\left(\overline{\mathbf{y}}_{c}-\overline{\mathbf{y}}\right)\left(\overline{\mathbf{y}}_{c}-\overline{\mathbf{y}}\right)^{T}$, and $\overline{\mathbf{y}}=\frac{1}{M} \sum_{m} \mathbf{y}_{m}$. The solution $\mathbf{V}_{l d a}=\arg \max _{\mathbf{V}} \frac{\left|\mathbf{V}^{T} \mathbf{S}_{B} \mathbf{V}\right|}{\left|\mathbf{V}^{T} \mathbf{S}_{W} \mathbf{V}\right|}=\left[\mathbf{v}_{1} \mathbf{v}_{2} \ldots \mathbf{v}_{H_{\mathbf{z}}}\right]$, where $\left\{\mathbf{v}_{h_{\mathbf{z}}}, h_{\mathbf{z}}=1, \ldots, H_{\mathbf{z}}\right\}$ is the set of generalized eigenvalues of $\mathbf{S}_{B}$ and $\mathbf{S}_{W}$ corresponding to the $H_{\mathbf{z}}(\leq C-1)$ largest generalized eigenvalues $\left\{\lambda_{h_{\mathbf{z}}}, h_{\mathbf{z}}=1, \ldots, H_{\mathbf{z}}\right\}: \mathbf{S}_{B} \mathbf{v}_{h_{\mathbf{z}}}=$ $\lambda_{h_{\mathbf{z}}} \mathbf{S}_{W} \mathbf{v}_{h_{\mathbf{z}}}, h_{\mathbf{z}}=1, \ldots, H_{\mathbf{z}}$. Thus, the discriminant feature vector $\mathbf{z}_{m}$ is obtained as: $\mathbf{z}_{m}=\mathbf{V}_{l d a}^{T} \mathbf{y}_{m}$, and a classifier can then be applied.

\section{EXPERIMENTAL RESULTS}

To evaluate the proposed framework, gait recognition experiments are carried out on the USF HumanID "Gait Challenge" data sets version 1.7 [1] for preliminary evaluation. The human gait sequences in these data sets were captured under different conditions (walking surfaces, shoe types and viewing angles). The gallery set contains 71 sequences (subjects) and seven experiments (probe sets) are designed for human identification. The capturing condition for each probe set is summarized in brackets after the probe name in Table 1, where $C, G, A, B, L, R$, standing for cement surface, grass surface, shoe type A, shoe type B, left view, and right view, respectively. The capturing condition of the gallery set is GAR. The silhouette data extracted by the baseline algorithm [1] is the input to the proposed algorithm for fair comparison against other gait recognition algorithms.

There are 731 gait samples obtained in the Gallery set and each subject has an average of roughly 10 samples available. The samples are normalized to a canonical size of $I_{1} \times I_{2} \times$ $I_{3}=128 \times 88 \times 20$. MPCA is then applied to extract features from the half cycles $\left(\left\{\mathcal{X}_{m}, m=1, \ldots, M\right\}\right)$ obtained from the gallery set, with $K=1$. The number of eigenvectors kept in each mode is: $P_{1}=61, P_{2}=42$ and $P_{3}=17$, determined by setting $Q^{(1)}=Q^{(2)}=Q^{(3)}=Q=0.97$, which captures approximately $92 \%$ of the total variations of the gallery gait samples in the projected tensor subspace. The 1-mode unfolding, i.e., concatenating the frames of a sequence sequentially in a row, of two gait samples and the mean gallery gait sample, are shown in Figs. 2(a) and 2(b), respectively.

The eigentensors obtained from the gallery gait sequences through MPCA are named EigenTensorGaits as in [7] and LDA is applied on selected EigenTensorGaits to extract features for recognition. The results reported below are obtained with $H_{\mathbf{y}}=170$, determined through empirical study. Figure 2(c) shows the 1-mode unfolding of seven discriminative EigenTensorGaits for illustration. From the figure, it is observed that the EigenTensorGaits act as a set of multiresolution filters, and the projection using them is very similar to applying a filter bank in multiresolution analysis. Each EigenTensorGait can be viewed as a filter and the projection is similar to a filtering process.

To measure the similarity of one test sample feature $\mathbf{z}$ against $N_{c}$ sample features $\mathbf{z}_{n_{c}}$ of a subject $c$, the principle of nearest neighbor classifier is applied. The matching score of $\mathbf{z}$ with subject $c$ is obtained as $S(\mathbf{z}, c)=-\min _{n_{c}} d\left(\mathbf{z}, \mathbf{z}_{n_{c}}\right)$. Such a simple classifier is selected to study the performance mainly contributed by the MPCA+LDA feature extraction algorithm although better classifiers can be investigated. The seven distance measures for vectors in [13] are tested and the best results are obtained with the Mahalanobis+angle distance (MAD) measure: $d(\mathbf{a}, \mathbf{b})=\frac{\sum_{h_{\mathbf{Z}}=1}^{H_{\mathbf{Z}}} \mathbf{a}\left(h_{\mathbf{z}}\right) \cdot \mathbf{b}\left(h_{\mathbf{z}}\right)}{\sqrt{\lambda_{h_{\mathbf{Z}}} \sum_{h_{\mathbf{z}}=1}^{H_{\mathbf{z}}} \mathbf{a}\left(h_{\mathbf{z}}\right)^{2} \sum_{h_{\mathbf{z}}=1}^{H_{\mathbf{Z}}} \mathbf{b}\left(h_{\mathbf{z}}\right)^{2}}}$, where $H_{\mathbf{z}}=C-1=70$ and $\lambda_{h_{\mathbf{z}}}$ is the generalized eigen- 


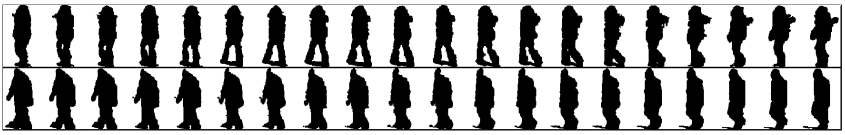

(a) 1-mode unfolding of two gait silhouette samples.

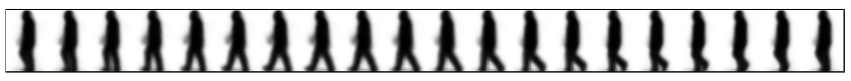

(b) 1-mode unfolding of the mean of the gallery gait silhouette samples.

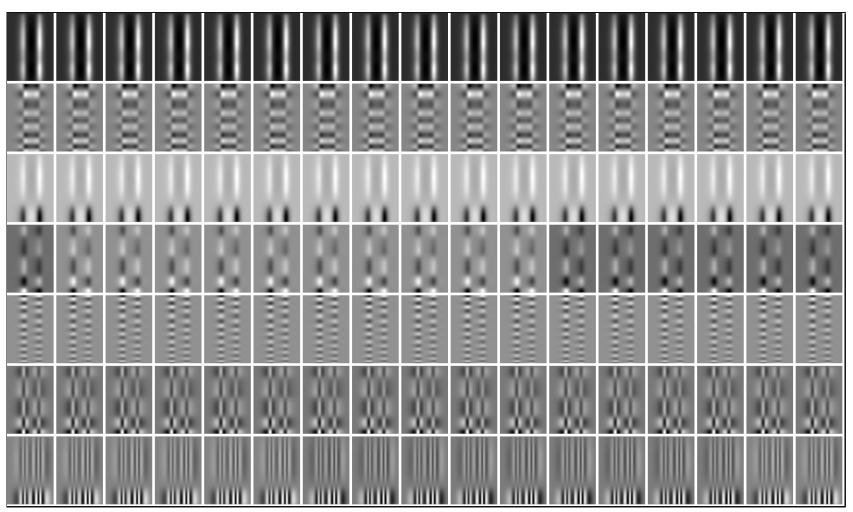

(c) The $1^{s t}, 15^{t h}, 30^{t h}, 45^{t h}, 60^{t h}, 75^{t h} \& 90^{t h}$ discriminative EigenTensorGaits.

Fig. 2. Illustration of gait samples and EigenTensorGaits.

value defined in previous section.

To obtain the matching score of a probe sequence $p$ with $N_{p}$ samples against a gallery sequence $g$ with $N_{g}$ samples, the approach in [6] is adopted, which proposed that the distance calculation process should be symmetric with respect to probe and gallery sequences. If the probe and gallery sequences were interchanged, the computed distance would be identical. The details are described as follows: each probe sample feature $\mathbf{z}_{n_{p}}$ is matched against the gallery sequence to obtain $S\left(\mathbf{z}_{n_{p}}, g\right)$ and each gallery sample feature $\mathbf{z}_{n_{g}}$ is matched against the probe sequence to obtain $S\left(\mathbf{z}_{n_{g}}, p\right)$. The matching score between the probe sequence $p$ and the gallery sequence $g$ is the sum of the mean matching score of $p$ against $g$ and that of $g$ against $p: S(p, g)=\frac{1}{N_{p}} \sum_{n_{p}=1}^{N_{p}} S\left(\mathbf{z}_{n_{p}}, g\right)+$ $\frac{1}{N_{g}} \sum_{n_{g}=1}^{N_{g}} S\left(\mathbf{z}_{n_{g}}, p\right)$. The identity of the gallery sequence with the highest matching score $S(p, g)$ is assigned to the probe sequence $p$.

The proposed algorithm is compared against the stateof-the-art gait recognition algorithms: the Hidden Markov Model (HMM) framework [3], the linear time normalization (LTN) algorithm [6], the Gait Energy Image (GEI) algorithm [14] and the MPCA-based algorithm using HOSVD truncation (MPCA-HT) [7]. The baseline (BL) algorithm [1] is also included for the sake of completeness. For the HMM algorithm, the direct approach is chosen for comparison. For the LTN algorithm, the LTN using the silhouette feature (LTN$\mathrm{S}$ ) is chosen for comparison. For the GEI method, the results involving the synthetic templates are not included for fair comparison. The identification performance is measured by the Cumulative Match Characteristic (CMC) [1], which plots identification rates $P_{I}$ within a given rank $k$ (rank $k$ results report the percentage of probe subjects whose true match in the gallery set was in the top $k$ matches). The detailed results are depicted using the CMCs in Fig. 4, and the comparison with the state-of-the-art algorithms is in Table 1, where the rank 1 and rank 5 identification rates are listed for each probe (A to $\mathrm{G}$ ) together with their averages. The best results for all the probe and rank combinations are highlighted by boldface font in the table. From the table, MPCA+LDA has achieved the best overall performance in both rank 1 and rank 5, compared against all the other algorithms, although with simple preprocessing, cycle partition and matching algorithms. A detailed discussion and further analysis can be found in [9].

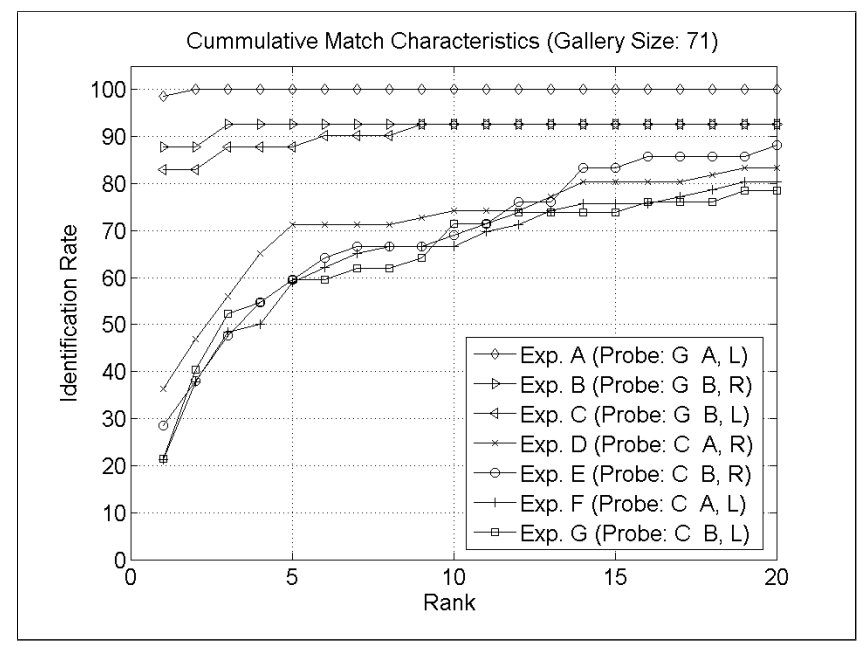

Fig. 3. CMC curves up to rank 20 for MPCA+LDA.

\section{CONCLUSIONS AND FUTURE WORKS}

This paper proposes a gait recognition algorithm using MPCA plus LDA. The new multilinear principal component analysis (MPCA) framework looks for a multilinear projection onto a tensor subspace of lower dimensionality that captures most of the variances in the original gait samples. MPCA is used to extract gait features from gait sequences in their natural tensor representation directly and LDA is applied on selected eigentensors afterwards for recognition. This MPCA+LDA approach for gait recognition is tested in the experiments and the results show that with a simple classifier (and simple preprocessing and cycle partition algorithms), the proposed method outperforms the state-of-the-art gait recognition algorithms. This MPCA-based approach is very promising for gait recognition and other tensor object recognition, and it can be generalized to the development of other tensor subspace algorithms in the future. 
Table 1. Comparison of gait recognition through MPCA+LDA and the state-of-the-art gait recognition algorithms.

\begin{tabular}{|c|c|c|c|c|c|c|c|c|c|c|c|c|}
\hline \multirow[b]{2}{*}{ Probe } & \multicolumn{6}{|c|}{$P_{I}(\%)$ at Rank 1} & \multicolumn{6}{|c|}{$P_{I}(\%)$ at Rank 5} \\
\hline & $\mathrm{BL}$ & HMM & LTN & GEI & MPCA-HT & MPCA+LDA & $\mathrm{BL}$ & HMM & LTN & GEI & MPCA-HT & MPCA+LDA \\
\hline A (GAL) & 79 & 99 & 94 & 100 & 94 & 99 & 96 & 100 & 99 & 100 & 99 & 100 \\
\hline $\mathrm{B}(\mathrm{GBR})$ & 66 & 89 & 83 & 85 & 76 & 88 & 81 & 90 & 85 & 85 & 83 & 93 \\
\hline $\mathrm{C}(\mathrm{GBL})$ & 56 & 78 & 78 & 80 & 66 & 83 & 76 & 90 & 83 & 88 & 81 & 88 \\
\hline $\mathrm{D}$ (CAR) & 29 & 35 & 33 & 30 & 27 & 36 & 61 & 65 & 65 & 55 & 64 & 71 \\
\hline $\mathrm{E}(\mathrm{CBR})$ & 24 & 29 & 24 & 33 & 36 & 29 & 55 & 65 & 67 & 55 & 52 & 60 \\
\hline $\mathrm{F}(\mathrm{CAL})$ & 30 & 18 & 17 & 21 & 15 & 21 & 46 & 60 & 58 & 41 & 53 & 59 \\
\hline $\mathrm{G}(\mathrm{CBL})$ & 10 & 24 & 21 & 29 & 19 & 21 & 33 & 50 & 48 & 48 & 48 & 60 \\
\hline Average & 42 & 53 & 50 & 54 & 48 & 54 & 64 & 74 & 72 & 67 & 68 & 76 \\
\hline
\end{tabular}

\section{APPENDIX: NOTATIONS AND BASIC MULTILINEAR ALGEBRA}

This work denotes vectors by lowercase boldface letters, e.g., $\mathbf{x}$; matrices by uppercase boldface, e.g., $\mathbf{U}$; and tensors by calligraphic letters, e.g., $\mathcal{A}$. Their elements are denoted with indices in brackets. Indices are denoted by lowercase letters and span the range from 1 to the uppercase letter of the index, e.g., $n=1,2, \ldots, N$. Throughout this paper, the discussion is restricted to real-valued vectors, matrices and tensors since the gait recognition application involves real data only.

An $N^{t h}$-order tensor is denoted as: $\mathcal{A} \in \mathbb{R}^{I_{1} \times I_{2} \times \ldots \times I_{N}}$. It is addressed by $N$ indices $i_{n}, n=1, \ldots, N$, and each $i_{n}$ addresses the $n$-mode of $\mathcal{A}$. The $n$-mode product of a tensor $\mathcal{A}$ by a matrix $\mathbf{U} \in \mathbb{R}^{J_{n} \times I_{n}}$, denoted by $\mathcal{A} \times_{n} \mathbf{U}$, is a tensor with entries: $\left(\mathcal{A} \times_{n} \mathbf{U}\right)\left(i_{1}, \ldots, i_{n-1}, j_{n}, i_{n+1}, \ldots, i_{N}\right)=$ $\sum_{i_{n}} \mathcal{A}\left(i_{1}, \ldots, i_{N}\right) \cdot \mathbf{U}\left(j_{n}, i_{n}\right)$. The scalar product of two tensors $\mathcal{A}, \mathcal{B} \in \mathbb{R}^{I_{1} \times I_{2} \times \ldots \times I_{N}}$ is defined as: $<\mathcal{A}, \mathcal{B}>=$ $\sum_{i_{1}} \sum_{i_{2}} \ldots \sum_{i_{N}} \mathcal{A}\left(i_{1}, i_{2}, \ldots, i_{N}\right) \cdot \mathcal{B}\left(i_{1}, i_{2}, \ldots, i_{N}\right)$ and the Frobenius norm of $\mathcal{A}$ is defined as $\|\mathcal{A}\|_{F}=\sqrt{\langle\mathcal{A}, \mathcal{A}\rangle}$. The " $n$-mode vectors" of $\mathcal{A}$ are defined as the $I_{n}$-dimensional vectors obtained from $\mathcal{A}$ by varying the index $i_{n}$ and keeping the other indices fixed. A rank- 1 tensor $\mathcal{A}$ equals to the outer product of $N$ vectors: $\mathcal{A}=\mathbf{u}^{(1)} \circ \mathbf{u}^{(2)} \circ \ldots \circ \mathbf{u}^{(N)}$, which means that $\mathcal{A}\left(i_{1}, i_{2}, \ldots, i_{N}\right)=\mathbf{u}^{(1)}\left(i_{1}\right) \cdot \mathbf{u}^{(2)}\left(i_{2}\right) \cdot \ldots \cdot \mathbf{u}^{(N)}\left(i_{N}\right)$ for all values of indices. Unfolding $\mathcal{A}$ along $n$-mode is denoted as $\mathbf{A}_{(n)} \in \mathbb{R}^{I_{n} \times\left(I_{1} \times \ldots \times I_{n-1} \times I_{n+1} \times \ldots \times I_{N}\right)}$, and the column vectors of $\mathbf{A}_{(n)}$ are the $n$-mode vectors of $\mathcal{A}$.

Any tensor $\mathcal{A}$ can be expressed as the product: $\mathcal{A}=\mathcal{S} \times{ }_{1}$ $\mathbf{U}^{(1)} \times{ }_{2} \mathbf{U}^{(2)} \times \ldots \times{ }_{N} \mathbf{U}^{(N)}$, where $\mathcal{S}=\mathcal{A} \times{ }_{1} \mathbf{U}^{(1)^{T}} \times_{2}$ $\mathbf{U}^{(2)^{T}} \ldots \times_{N} \mathbf{U}^{(N)^{T}}$, and $\mathbf{U}^{(n)}=\left(\mathbf{u}_{1}^{(n)} \mathbf{u}_{2}^{(n)} \ldots \mathbf{u}_{I_{n}}^{(n)}\right)$ is an orthogonal $I_{n} \times I_{n}$ matrix. The decomposition can also be written as the sum of $\prod_{n=1}^{N} I_{n}$ rank-1 tensors:

$$
\mathcal{A}=\sum_{i_{1}=1}^{I_{1}} \ldots \sum_{i_{N}=1}^{I_{N}} \mathcal{S}\left(i_{1}, \ldots, i_{N}\right) \mathbf{u}_{i_{1}}^{(1)} \circ \ldots \circ \mathbf{u}_{i_{N}}^{(N)} .
$$

\section{REFERENCES}

[1] S. Sarkar, P. J. Phillips, Z. Liu, I. Robledo, P. Grother, and K. W. Bowyer, "The human ID gait challenge problem: Data sets, performance, and analysis," IEEE Trans. Pattern Anal. Machine Intell., vol. 27, no. 2, pp. 162-177, Feb. 2005.

[2] N. V. Boulgouris, D. Hatzinakos, and K. N. Plataniotis, "Gait recognition: a challenging signal processing technology for biometrics," IEEE Signal Processing Mag., vol. 22, no. 6, Nov. 2005.

[3] A. Kale, A. N. Rajagopalan, A. Sunderesan, N. Cuntoor, A. Roy-Chowdhury, V. Krueger, and R. Chellappa, "Identification of humans using gait," IEEE Trans. Image Processing, vol. 13, no. 9, pp. 1163-1173, Sept. 2004.

[4] G. Shakhnarovich and B. Moghaddam, "Face recognition in subspaces," in Handbook of Face Recognition, Stan Z. Li and Anil K. Jain, Eds. 2004, pp. 141-168, Springer-Verlag.

[5] M. H. C. Law and A. K. Jain, "Incremental nonlinear dimensionality reduction by manifold learning," IEEE Trans. Pattern Anal. Machine Intell., vol. 28, no. 3, pp. 377-391, Mar. 2006.

[6] N. V. Boulgouris, K. N. Plataniotis, and D. Hatzinakos, "Gait recognition using linear time normalization," Pattern Recognition, vol. 39, no. 5, pp. 969-979, 2006.

[7] H. Lu, K.N. Plataniotis, and A.N. Venetsanopoulos, "Multilinear principal component analysis of tensor objects for recognition," in Proc. Int. Conf. on Pattern Recognition, August 2006.

[8] L. De Lathauwer, B. De Moor, and J. Vandewalle, "A multilinear singualr value decomposition," SIAM Journal of Matrix Analysis and Applications, vol. 21, no. 4, pp. 1253-1278, 2000.

[9] H. Lu, K.N. Plataniotis, and A.N. Venetsanopoulos, "MPCA: Multilinear principal component analysis of tensor objects," IEEE Trans. Neural Networks, submitted 2006 (under review).

[10] P. Kroonenberg and J. Leeuw, "Principal component analysis of three-mode data by means of alternating least squares algorithms," Psychometrika, vol. 45, no. 1, pp. 69-97, 1980.

[11] J. Wang, K. N. Plataniotis, and A. N. Venetsanopoulos, "Selecting discriminant eigenfaces for face recognition," Pattern Recognition Letters, vol. 26, no. 10, pp. 1470-1482, 2005.

[12] P. N. Belhumeur, J. P. Hespanha, and D. J. Kriegman, "Eigenfaces vs. fisherfaces: Recognition using class specific linear projection," IEEE Trans. Pattern Anal. Machine Intell., vol. 19, no. 7, pp. 711-720, July 1997.

[13] H. Moon and P. J. Phillips, "Computational and performance aspects of pca-based face recognition algorithms," Perception, vol. 30, pp. 303-321, 2001.

[14] J. Han and B. Bhanu, "Individual recognition using gait energy image," IEEE Trans. Pattern Anal. Machine Intell., vol. 28, no. 2, pp. 316-322, Feb. 2006. 